\title{
Meta
}

Journal des traducteurs

Translators' Journal

\section{Ellis, Roger and Ruth Evans (1994): The Medieval Translator (4), Exeter, University of Exiter, 256 p.}

\section{Michel Ballard}

Volume 42, numéro 3, septembre 1997

L’interprétation en langues des signes

URI : https://id.erudit.org/iderudit/001959ar

DOI : https://doi.org/10.7202/001959ar

Aller au sommaire du numéro

Éditeur(s)

Les Presses de l'Université de Montréal

ISSN

0026-0452 (imprimé)

1492-1421 (numérique)

Découvrir la revue

Citer ce compte rendu

Ballard, M. (1997). Compte rendu de [Ellis, Roger and Ruth Evans (1994): The

Medieval Translator (4), Exeter, University of Exiter, 256 p.] Meta, 42(3),

586-589. https://doi.org/10.7202/001959ar

Ce document est protégé par la loi sur le droit d'auteur. L'utilisation des services d'Érudit (y compris la reproduction) est assujettie à sa politique d'utilisation que vous pouvez consulter en ligne.

https://apropos.erudit.org/fr/usagers/politique-dutilisation/
Cet article est diffusé et préservé par Érudit.

Érudit est un consortium interuniversitaire sans but lucratif composé de l'Université de Montréal, l'Université Laval et l'Université du Québec à Montréal. Il a pour mission la promotion et la valorisation de la recherche. https://www.erudit.org/fr/ 
- ELLIS, Roger and Ruth EVANS (1994): The Medieval Translator (4), Exeter, University of Exeter, $256 \mathrm{p}$.

Ce volume rassemble les Actes du troisième colloque de ce type, qui s'est tenu à Cardiff en 1991.

Après la table des matières et les notices bio-bibliographiques concernant les auteurs, on trouve une introduction bicéphale rédigée par les organisateurs du colloque. Roger Ellis a réalisé la traditionnelle (mais ô combien utile!) présentation ordonnée des différentes communications. Ruth Evans avec son essai sur «Translating Past Cultures?» s'efforce d'offrir une mise en perspective (et même en prospective) des études sur la traduction médiévale. Cette réflexion érudite fait intervenir les grands classiques modernes et anciens de la traductologie, de la narratologie et de la sémiotique, et de la traduction médiévale. Elle invite les spécialistes de cette dernière discipline à élargir leur approche en intégrant l'apport de certaines démarches: la traduction comme métaphore de l'étrangéité, de l'interface; le discours féministe; etc. Il s'agit de se donner des outils conceptuels pour explorer le thème du dialogue entre les cultures, pour éclaircir la traduction médiévale de l'intérieur et permettre de la situer dans le cadre plus général des études traductologiques. L'ensemble aurait pu être plus fortement structuré, on a parfois l'impression d'un collage de notes de lecture. Mais, il faut reconnaître qu'il y a là un effort de réflexion et une incitation à l'innovation qui ne peuvent laisser indifférent; par ailleurs, même les 
non-spécialistes apprécieront les nombreuses références et notes de lecture concernant des chercheurs anglo-saxons qui ne sont pas toujours connus du public universitaire moyen et francophone.

L'article de Jocelyn Wogan-Browne est intéressant à plus d'un titre : elle aborde un thème qui va réapparaître à plusieurs reprises dans ce volume (féminité et traduction) et ceci dans le cadre d'un genre (les écrits hagiographiques) et d'une localisation géographicosocio-religieuse (les monastères féminins de Barking et de Chatteris). Elle examine un corpus de vies de saints traduites à la fin des $\mathrm{XII}^{\mathrm{e}}$ et $\mathrm{XIII}^{\mathrm{e}}$ siècles en français, langue de culture dans les institutions monastiques, et s'efforce d'en dégager des stratégies spécifiques qui sont parfois à la limite de la traduction mais laissent de toute évidence paraître une conscience féministe dans les paratextes (prologues) aussi bien que des choix et des infléchissements révélateurs au niveau de la réécriture.

La contribution de Rina Drory est nerveuse, bien documentée et clairement exposée. Elle se concentre à la fois sur un genre, le maqamat (sorte de roman picaresque apparu dans la littérature arabe à la fin du $\mathrm{X}^{\mathrm{e}}$ siècle) et un traducteur-auteur juif, Judah al-Harizi (1 170 1235). Né à Tolède, ce Du Bellay de la diaspora, traduisit le maqamat en Espagne du Nord ou en Provence avant de composer son propre Maqamat en Orient pour les juifs d'Orient. L'article de Rina Drory examine avec beaucoup de brio les motivations et la manière d'al-Harizi, tout en nous offrant des traductions de préfaces rares et éclairantes.

La féminité est présente à nouveau dans l'intervention d'Helen Philipps mais cette fois comme métaphore et pivot narratologique. Le corpus de base est la Complaint of Venus de Chaucer qui est en partie traduite d'une sélection de balades écrites par Oton de Grannson (c. 1340-1397). Avec une grande minutie, Helen Philipps examine les transformations que Chaucer a fait subir au texte de Grannson, ne serait-ce que parce qu'il a opté pour un locuteur féminin. Helen Philipps étend ensuite la métaphore féminine de la traduction avec tout ce qu'elle suppose de caractéristiques aux moments où Chaucer laisse paraître la main du traducteur dans son œuvre. Il y a là une démarche intéressante, qui mériterait d'être vérifiée sur un autre corpus s'il en est qui s'y prête.

Ian Johnson conteste une assertion de Genette selon laquelle le paratexte est beaucoup moins important au Moyen Âge qu'aujourd'hui. Il en donne pour exemple ceux d'Osbern Bokenham, hagiographe du milieu du XV' $\mathrm{XV}^{\mathrm{e}}$ siècle. Le prologue de ses Legendys of Hooly Women révèle un traducteur soucieux d'établir son statut sur un pied d'égalité avec l'auteur grâce à la grille scholastique des Quatres Causes. Le prologue contient également des détails autobiographiques sur les circonstances de la traduction, sa genèse, les impulsions ou le soutien que le traducteur a reçus d'amis ou de mécènes. Souvent aussi derrière ces détails circonstanciés perce une stratégie laissant paraître des inquiétudes et le souci d'asseoir son autorité.

C'est un cas d'autotraduction que Mary-Jo Arn étudie avec Charles d'Orléans. Le point d'interrogation qui ponctue ce titre est lié au fait que non seulement ce poète français a modifié son texte pour des raisons linguistiques mais s'est aussi laissé entraîner à un travail de réécriture (ou de poursuite de l'écriture) en langue anglaise. Travail très soigné et précis de cette brève intervention qui bénéficie des recherches que l'auteur a antérieurement réalisées pour son édition critique du même poète.

On ne peut que louer Veronica Lawrence pour le sérieux de sa démarche qui repose sur le comparatisme. L'auteur dont elle traite est Richard Whitford (1475-1542) et chez ce religieux, auteur de nombreuses traductions, elle sélectionne celles dont on possède les originaux afin de pouvoir mener une étude serrée de ses techniques de traduction. Cette utilisation du commentaire de traduction, perceptible dans nombre des interventions de ce recueil, est indéniablement la marque d'une démarche scientifique authentique. Cette rigoureuse étude est étendue aux textes plus personnels de Richard Whitford où sont 
intégrés des passages de l'écriture. Certains des traits relevés dans Richard Whitford seraient à rapprocher de ceux qui sont perceptibles chez certains écrivains et traducteurs de la même époque en France (cf. Ballard 1994: 97-101).

La comparaison de manuscrits et de textes demeure au centre de la stratégie de la communication suivante mais c'est à un genre différent qu'elle s'applique: L'Itinerius de Johannes Witte de Hese, comme modèle de récit de voyage au $X V^{\mathrm{e}}$ siècle. L'étude de Scott $\mathrm{D}$. Westrem se développe en trois mouvements : résumé du contenu du livre; contexte général des récits de voyage à la fín du Moyen Âge; enfin comparaison de différents états du texte latin et de ses traductions en hollandais. Tout autant qu'une étude de traduction, il s'agit d'une exploration des transformations de l'original sous ses diverses formes manuscrites et imprimées.

«The translation of the Feminine. Untranslatable Dimensions of the Anchoritic works» d'Anne Savage, sur un mode très personnel et dans un registre différent, aborde le thème annoncé par l'introduction de Ruth Evans et que l'on retrouvera dans l'intervention de Tixier, à savoir le texte médiéval en traduction pour un public moderne. Mais ici cette exploration se double d'un élargissement de la notion de traduction: Anne Savage s'interroge sur la part irréductible d'étrangeté que comportent les textes destinés aux femmes anachorètes pour une lectrice ou un lecteur moderne et ceci est l'occasion de dresser un tableau de leur condition et des schémas spirituels qui la constituent et au cour desquels on retrouve la traduction comme métaphore du passage d'un corps féminin asservi dans le mariage terrestre à un corps féminin libéré dans la joie du mariage céleste. Cette démarche donne une communication qui semble plus documentaire (ou militante) que traductologique.

C'est tout autant un travail d'exploration herméneutique que de recherche en expression par l'écriture et la traduction que nous livre Denis Renevey. Sa base d'étude est le commentaire qu'a réalisé Richard Rolle du début du Cantique des cantiques où il joue ce rôle fondamental de relais entre les non-initiés et le message divin tout en utilisant son interprétation pour transmettre sa propre expérience mystique.

Comme l'écrit fort justement Roger Ellis dans son introduction, l'intervention de René Tixier vient clore avec bonheur l'ensemble de ce colloque, même si l'on est en droit de se demander si son insertion ne relève pas un peu du subterfuge: le titre du colloque est «Le traducteur médiéval», or il n'y a pas de traducteur médiéval dans cette intervention mais l'examen de deux traducteurs modernes d'un texte médiéval. Le texte de départ est «The Cloud of Unknowing», poème mystique anonyme du XIV siècle, qui a servi de base à la thèse de René Tixier. Il examine avec beaucoup de compétence et de brio les deux (premières) traductions qui en ont été réalisées au $\mathrm{XX}^{\mathrm{e}}$ siècle: celle de Dom Maurice Noetinger, moine de Solesmes exilé en Angleterre, qui parut en 1925; et celle d'Armel Guerne «parue en 1953 aux Cahiers du Sud» (p. 218). L'analyse est organisée autour de dix concepts clés et fait intervenir également les traductions anglaises de ce texte. Les deux traductions appartiennent à deux traditions différentes et très typées: celle de Dom Maurice Noetinger «est beaucoup plus qu'une simple traduction: c'est en réalité un travail d'explication, d'expansion, de description et d'interprétation du texte original» (p. 219); celle d'Armel Guerne est «une traduction souvent plus littérale, plus concrète, aux images plus heurtées et aux constructions plus elliptiques» (p. 219) à cela s'ajoute l'utilisation d'«un français ancien reconstitué, dont le pseudo-archaïsme pourrait finir par constituer un obstacle» (ibid). On trouve donc réunies ici les caractéristiques de deux grandes traditions de la traduction mêlées aux problèmes de la traduction diachronique, nombre des choix des deux traducteurs français se retrouvent dans les traductions intralinguales qui sont évoquées en illustration de cet examen serré de deux options fondamentales. René Tixier nous livre là une étude très sérieuse, nourrie de tout son passé de recherche, et qui fait apparaître l'importance du commentaire comparé en traductologie. 
Le volume s'achève sur une bibliographie sélective de trois pages et demie et sur un index des noms propres et de certains textes examinés ( 6 pages sur 2 colonnes).

On peut se demander pourquoi cette bibliographie est sélective et quels ont été les critères de sélection. C'est ainsi que l'on y retrouve les grands classiques comme Walter Benjamin, Georges Steiner (mais pas T. R. Steiner), Nida, L. G. Kelly, ou même H. Belloc alors que par exemple H. Kittel et A. P. Frank (p. 19), Eva Hoffman, Jonathan Rutherford, Paul Chavy (p. 37) ou Manaranche (p. 245), qui sont mentionnés dans divers textes, ne sont pas repris dans la bibliographie. C'est dommage, étant donné les abondantes références que contiennent les interventions. Peut-être aurait-on pu s'en tenir tout au moins à la solution classique, mais pratique, laissant le soin à chaque auteur de donner sa propre bibliographie à la fin de son article. Peut-être aussi y aurait-il eu moyen d'alléger le système de notes, qui est un peu lourd; certaines auraient pu être transformées en simple, références entre parenthèses dans le texte, tandis que l'on aurait pu faire un effort pour intégrer le contenu des autres dans le texte.

Ce volume constitue un apport indéniable à la recherche sur la traduction médiévale, qui sera sans doute apprécié autant par les spécialistes que par ceux qui, de manière générale, s'intéressent à l'histoire de la traduction. Les communications les plus convaincantes sont celles qui font intervenir la comparaison de textes mais elles sont toutes richement documentées, souvent assises sur la recherche principale de conférenciers ou des contributions antérieures. On a l'impression d'une réflexion vivante, ouverte aux derniers développements des champs envisagés et qui, souvent, s'efforce d'instaurer des points de vue originaux et de décloisonner l'approche historique de son matériau. Il convient de remercier Roger Ellis et Ruth Evans pour avoir su susciter et organiser ce livre dont la présentation matérielle fait également honneur aux Presses de l'Université d'Exeter.

MICHEL BALLARD

Université d'Artois, Arras, France

\section{RÉFÉRENCE}

BALLARD, Michel (1994): De Cicéron à Benjamin. Traducteurs, traductions réflexions, $2^{\mathrm{e}}$ édition revue et corrigée (1 ${ }^{\text {re }}$ éd. 1992), Lille, P.U.S. 